\title{
Frequência de retinopatia da prematuridade em recém-nascidos no Hospital das Clínicas da Faculdade de Medicina de Ribeirão Preto da Universidade de São Paulo
}

\author{
Frequency of retinopathy of prematurity at newborns at the Clinical Hospital, \\ Ribeirão Preto Medical School, University of São Paulo
}

\author{
Rogério Neri Shinsato \\ Letícia Paccola ${ }^{2}$ \\ Walusa Assad Gonçalves ${ }^{3}$ \\ JoséCarlos Barbosa ${ }^{4}$ \\ Francisco Eulógio Martinez ${ }^{5}$ \\ Maria de Lourdes Veronese Rodrig'ues ${ }^{6}$ \\ Rodrigo Jorg'e $^{7}$
}

\begin{tabular}{|l|}
\hline RESUMO \\
\hline Objetivos: Determinar a frequência de retinopatia da prematuridade \\
no Hospital das Clínicas da Faculdade de Medicina de Ribeirão Preto \\
(HCFMRP-USP) e verificar a associação da retinopatia da prematuridade \\
com fatores de risco conhecidos. Métodos: Foi realizada análise pros- \\
pectiva de 70 pacientes, nascidos no HCFMRP-USP, com peso inferior \\
a 1.500 gramas, no período de um ano. Os pacientes foram divididos em \\
dois grupos (Retinopatia da prematuridade e Normal) para realização de \\
análise estatística com relação a fatores de risco conhecidos. Adotou-se \\
nível de significância de 5\%. Resultados: A frequência de retinopatia da \\
prematuridade foi de 35,71\% entre os pré-termos estudados. Os fatores \\
pesquisados que apresentaram relação de risco para o desenvolvimento \\
da doença foram: peso (p=0,001), idade gestacional (p=0,001), escore \\
SNAPPE II (p=0,008), uso de oxigenoterapia por intubação (p=0,019) \\
e por pressão positiva de vias aéreas (p=0,0017), múltiplas transfusões \\
sanguíneas (p=0,01) e uso de diuréticos (p=0,01). Conclusão: A fre- \\
quência de retinopatia da prematuridade foi de 35,71\% entre os pré- \\
termos nascidos com menos de 1.500 g. Vários fatores de risco foram \\
identificados nos recém-nascidos do HCFMRP-USP, sendo constatado \\
que crianças mais pré-termos apresentam formas mais graves de retinopatia \\
da prematuridade.
\end{tabular}

Descritores: Retinopatia da prematuridade/epidemiologia; Recém-nascido; Fatores de risco; Estudos retrospectivos; Idade gestacional; Peso ao nascer

\section{INTRODUÇÃO}

A retinopatia da prematuridade (ROP) é uma doença multifatorial que afeta a vascularização da retina de recém-nascidos pré-termos. O termo retinopatia da prematuridade veio substituir o termo fibroplasia retrolental descrita por Terry em $1942^{(1)}$, que relatou a presença de um tecido vascularizado retrolental em uma criança prematura.

Nos Estados Unidos, em 1953, a ROP foi responsável por 7.000 casos de cegueira $^{(2)}$. O uso controlado de oxigênio pelos médicos fez com que a proporção de cegueira causada por ROP, somente nos Estados Unidos, caísse de $50 \%$ em 1950 para $4 \%$ em $1960^{(3)}$. Atualmente, a incidência de retinopatia da prematuridade nos Estados Unidos é de $0,12 \%$ do total de 
nascimentos ou, um caso para cada 820 recém-nascidos ${ }^{(4)}$ e estima-se 300 novos casos de cegueira por ROP neste país por ano ${ }^{(5)}$.

No início da década de 1990 houve um grande aumento do número de deficientes visuais por ROP nos países em desenvolvimento. Isto ocorreu porque a melhora da qualidade dos serviços neonatais, com consequente aumento da sobrevida de pré-termos, não foi acompanhada por acesso destes a cuidados oftalmológicos ${ }^{(6-7)}$.

No Brasil, alguns estudos apontam um aumento dos casos de ROP, principalmente em grandes centros ${ }^{(8-9)}$. Estima-se que 16.000 recém-nascidos apresentem ROP anualmente sendo que $10 \%$ destes podem ficar cegos caso não sejam tratados ${ }^{(10)}$. Entretanto, esses dados não são exatos e somente em 2007 foram criadas as diretrizes brasileiras para a $\mathrm{ROP}^{(11)}$.

Vários fatores de risco vêm sendo associados ao desenvolvimento da ROP. O peso ao nascimento, a idade gestacional e uso de oxigenoterapia são exemplos já consagrados na literatura $^{(8,12-14)}$.

Em estudo retrospectivo realizado no HCFMRP-USP, foram considerados fatores de risco para a ROP: uso de fototerapia, múltiplas transfusões sanguíneas, presença de hemorragia intracraniana e índice de Apgar baixo no primeiro minuto ${ }^{(15)}$.

Devido à escassez de dados epidemiológicos relativos a essa doença no Brasil, optou-se por estudar prospectivamente os recém-nascidos pré-termos do Hospital das Clínicas da Faculdade de Medicina de Ribeirão Preto da Universidade de São Paulo (HCFMRP-USP), com o intuito de verificar: 1. Frequência da retinopatia nos recém-nascidos pré-termos do HCFMRP-USP no período de 1 ano; 2. Verificar a associação de ROP com os fatores de risco conhecidos.

\section{MÉTODOS}

Foi realizada análise prospectiva dos pacientes pré-termos de baixo peso (menor que 1.500 gramas), que nasceram no HCFMRP-USP, no período de 1 de fevereiro de 2005 a 31 de janeiro de 2006 e foram acompanhados no ambulatório de retinopatia da prematuridade do Setor de Retina e Vítreo do HCFMRP-USP.

Todos os pacientes foram encaminhados pelo serviço de pediatria do HCFMRP-USP seguindo os seguintes critérios: peso ao nascimento igual ou menor que 1.500 gramas.

Todos os pacientes foram avaliados entre a quarta e sexta semana de vida. O exame foi feito com oftalmoscopia indireta com lente de 28 dioptrias e uso de indentador após midríase medicamentosa com tropicamida 0,5\% (Mydriacyl ${ }^{\circledR}$, Alcon) e anotado na ficha do paciente seguindo a classificação internacional da doença de $1987^{(16)}$. Caso o pré-termo estivesse internado no momento do exame, este era avaliado na unidade neonatal do HCFMRP-USP.

O seguimento dos pacientes era realizado conforme o estágio da doença: pacientes com vascularização incompleta da retina eram acompanhados em intervalos de uma a três semanas até completar a vascularização; recém-nascido com ROP grau I, II ou III (excluindo doença limiar): acompanhamento semanal até a completa vascularização da retina; em pacientes com doença limiar era indicado tratamento com fotocoagulação ou crioterapia de retina; recém-nascido com grau IV: avaliação de explante escleral associado ou não a fotocoagulação ou crioterapia ou vitrectomia posterior; recém-nascido com grau V: deveria ser discutida a possibilidade de tratamento cirúrgico.

As crianças que não apresentaram nenhum grau de retinopatia da prematuridade foram consideradas não portadoras de ROP e incluídas no grupo Normal. Já as crianças que apresentaram algum grau de ROP foram consideradas do grupo ROP. O grau de retinopatia atribuído a cada paciente foi o mais grave verificado nos olhos do recém-nascido estudado. Foram pesquisados diversos fatores de risco para a ROP. Quanto às características do recém-nascido avaliou-se a idade gestacional (utilizando a data da última menstruação como primeira opção, seguido pela data por ultrassom e pelo método de Ballard), peso ao nascimento, presença de gestações múltiplas, índice de Apgar no primeiro e quinto minutos e escore SNAPPE II. Quanto às terapêuticas utilizadas considerou-se, o uso de oxigenoterapia em capela, por pressão positiva nas vias aéreas (CPAP) ou por ventilação mecânica, uso de indometacina, uso de surfactante, de aminofilina, de fototerapia, de múltiplas transfusões sanguíneas, de diurético e uso de corticosteróide (antenatal). Quanto às patologias detectadas durante a internação registrou-se a presença de desconforto respiratório inicial, de pneumotórax, de broncodisplasia (uso de oxigênio por 28 dias ou mais), de sepse (diagnóstico clínico ou laboratorial), de persistência do canal arterial (diagnóstico por ecocardiografia) e de hemorragia intracraniana (ultrassonografia transfontanelar entre 5 e 10 dias de vida).

Os dados foram coletados semanalmente e cadastrados em planilha do programa Excel da Microsoft. Para análise estatística, os pacientes foram divididos em dois grupos: Normal e ROP. Foi utilizado o teste exato de Fisher para variáveis categóricas, Teste F (ANOVA) para variáveis quantitativas e teste de Tukey de comparações múltiplas entre as médias. Adotou-se nível de significância de $\mathrm{p}<0,05$. Após a análise univariada para possíveis fatores de risco foi realizado um modelo de regressão logística múltipla para todos os fatores com $\mathrm{p}>0,1$.

\section{RESULTADOS}

No período de fevereiro de 2005 a janeiro de 2006 nasceram 92 crianças com peso inferior a 1.500 gramas, sendo que 19 evoluíram para óbito antes da primeira avaliação e três receberam alta e não retornaram para o exame. Desta forma foram avaliados 70 recém-nascidos.

A amostra foi dividida em dois grupos: ROP (grupo dos pacientes que apresentaram algum grau de retinopatia da prematuridade) e Normal (grupo dos pacientes que não apresentaram retinopatia da prematuridade). 


\begin{tabular}{|c|c|c|c|}
\hline Idade gestacional & 1,39 & $0,99-1,95$ & 0,058 \\
\hline Uso de ventilação mecânica & 0,44 & $0,09-2,16$ & 0,310 \\
\hline Múltiplas transfusões sanguíneas & 0,70 & $0,07-7,04$ & 0,750 \\
\hline Uso de diuréticos & 1,76 & $0,17-18,28$ & 0,630 \\
\hline Hemorragia intracraniana & 0,42 & $0,09-1,99$ & 0,270 \\
\hline Presença de sepse & 0,94 & $0,13-6,67$ & 0,950 \\
\hline
\end{tabular}

zenove crianças morreram $(20,6 \%)$ com peso inferior a 1.500 gramas e apenas três crianças não compareceram ao ambulatório. A taxa de mortalidade do serviço está, portanto, bem abaixo da nacional que é de $60 \%$ para crianças com esse peso ${ }^{(17)}$.

A incidência de retinopatia da prematuridade neste estudo foi de $35,7 \%$ considerando todos os pré-termos avaliados. $\mathrm{Na}$ literatura nacional é descrito incidências de $29,1 \%{ }^{(8)} ; 28,5 \%{ }^{(10)}$ e $27,2 \%{ }^{(13)}$ de ROP.

Todos estes estudos se situam numa faixa de incidência semelhante e apresentam uma frequência maior de ROP no estágio I, já este estudo apresentou uma frequência maior de ROP no estágio III. Isso poderia justificar o aumento da frequência de ROP encontrada. A menor frequência de ROP no estagio I neste trabalho pode significar uma falha na diferenciação deste estágio no exame oftalmológico de triagem.

O estudo CRYO-ROP apresentou uma incidência de $65,8 \%{ }^{(18)}$, entretanto o seu critério de inclusão quanto ao peso era menor que neste estudo ( $\mathrm{P} \leq 1.250$ gramas). Outro importante estudo americano, o ETROP, também incluiu RN com peso inferior a 1.250 gramas e mostrou uma incidência de $68 \%{ }^{(19)}$. Adequando este estudo a esse critério, encontra-se uma incidência de 46,8\%. A maior taxa de sobrevida de RN com peso inferior a 1.250 gramas nestes trabalhos até a primeira avaliação (4 semanas de vida) pode explicar uma maior prevalência de ROP nestes estudos ${ }^{(17-19)}$.

Devido à dificuldade de se pesquisar a idade gestacional, por ser imprecisa e desconhecida às vezes, optou-se por adotar somente o peso como critério de inclusão no estudo. Além disso, o uso somente do peso é prático para a triagem dos recém-nascidos e as diretrizes brasileiras de triagem da ROP, publicadas em $2007^{(11)}$ definem como critério de inclusão peso e/ ou idade gestacional $<32$ semanas. Por fim, baixa idade gestacional e baixo peso ao nascimento estão associados e têm uma mesma consequência: imaturidade do tecido retiniano ${ }^{(13-14,20-21)}$.

De fato, este estudo mostrou uma associação significativa de baixo peso e pequena idade gestacional com o desenvolvimento de ROP. Vários trabalhos na literatura demonstram que, quanto menor o peso e menor a idade gestacional, maior a chance de desenvolver $\operatorname{ROP}^{(13-14,20-21)}$. O baixo peso e a menor idade gestacional também estão associados com desenvolvimento de formas mais graves de ROP. No presente estudo, o estágio III da doença foi o mais frequente com $15,71 \%$ dos casos, se considerados os $\mathrm{RN}$ com peso $\leq 1.500 \mathrm{~g}$. Esse valor sobe para $23,91 \%$ se analisarmos somente recém-nascidos com peso $\leq 1.250 \mathrm{~g}$. Esses achados estão de acordo com os encontrados no CRYO-ROP e ETROP, que apresentaram aumento nas formas mais graves de ROP em recém-nascidos com peso menor e mais pré-termos. Analisando a média do peso e da idade gestacional entre os diferentes estágios de ROP, neste estudo, nota-se diferença estatisticamente significante entre o estágio III de ROP e o grupo normal (Teste de Tukey), confirmando a associação inversa entre a imaturidade retiniana e o estágio de ROP.

Foram tratados $10 \%$ dos casos com ROP, Lima, em um estudo anterior apresentou uma incidência de tratamento de $7,8 \%$. Este aumento pode ser justificado pelo aumento de casos no estágio III $^{(15)}$.

Atualmente, tem se questionado uma associação de risco com o escore de gravidade SNAPPE II e a retinopatia da prematuridade $^{(22)}$. Este estudo demonstrou esta associação $(p=0,008)$.

$\mathrm{O}$ uso de oxigenoterapia, neste estudo foi avaliado em dias e por aparelhos. A ventilação mecânica e o CPAP foram as formas que apresentaram diferenças estatisticamente significativas entre os grupos Normal e ROP $(p=0,0190$ e $p=0,0017$ respectivamente). Outros estudos comprovam a associação de risco para ROP com o uso de ventilação mecânica ${ }^{(13,20-21)}$ e $\mathrm{CPAP}^{(8)}$. Quanto mais imatura a criança maior a necessidade do uso de ventilação mecânica e CPAP, justificando este achado.

O uso de diuréticos e corticóides são formas de tratamento para a broncodisplasia pulmonar. Este estudo mostrou associação de risco para a ROP com o uso de diuréticos $(\mathrm{p}=0,01)$. No estudo multicêntrico STOP-ROP ${ }^{(23)}$ foi verificado que o uso de suplementação de oxigenoterapia em pré-termos com doença pré-limiar eleva o risco de doenças crônicas pulmonares e aumenta o uso de diuréticos e dias de internação. Estes achados poderiam justificar parcialmente esta associação, pois em nosso estudo ocorreu um aumento da frequência do estágio III da doença (mais grave), entretanto, não foi pesquisado o estágio pré-limiar definido no STOP-ROP especificamente. O uso de corticóides antenatal também não mostrou significância estatística $(\mathrm{p}=0,40)$, diferindo de outros trabalhos ${ }^{(24)}$. $\mathrm{O}$ fato de apresentar uma amostra com apenas 70 pacientes pode ter prejudicado esta associação. 
A presença de sepse neste estudo não foi considerada um fator de risco para a ROP, entretanto mostrou uma tendência à significância $(\mathrm{p}=0,052)$. Na literatura, encontra-se descrita esta associação ${ }^{(25)}$. Outro trabalho demonstrou que a sepse pode ser considerada como um fator de risco importante para triagem de ROP em pré-termos com peso entre $1.501 \mathrm{~g}$ a $2.000 \mathrm{~g}^{(26)}$. Entretanto, este trabalho apresenta uma amostra de tamanho reduzido, que pode ter prejudicado esta associação de risco.

Outro fator de risco que apresentou relação significativa entre os grupos foi a presença de múltiplas transfusões sanguíneas $(\mathrm{p}=0,01)$. Este achado também foi encontrado por vários autores na literatura ${ }^{(10,13,21)}$. Acredita-se que como a hemoglobina fetal tem maior afinidade ao oxigênio em relação à hemoglobina do adulto, a transfusão de hemoglobina do adulto geraria uma possível hiperóxia devido à maior liberação de oxigênio para os tecidos ${ }^{(27)}$. Outra teoria seria o aumento de radicais livres após transfusões, devido ao aumento do ferro livre plasmático ${ }^{(28)}$.

O modelo de regressão logística múltipla apresentou tendência à significância para a idade gestacional e os demais fatores de risco não apresentaram diferença estatisticamente significativa. A regressão logística leva em conta a contribuição relativa dos diversos fatores e deixa evidente que o verdadeiro fator de risco é a baixa idade gestacional e a consequente imaturidade dos diversos tecidos do RN. Em outras palavras, o verdadeiro fator de risco é a prematuridade e os outros fatores (baixa score SNAPPE ao nascimento, broncodisplasia, uso de oxigênio por ventilação mecânica, uso de diurético e necessidade de múltiplas transfusões) seriam apenas consequência desta prematuridade e impulsionados pela mesma em direção à significância estatística verificada nas análises isoladas.

\section{CONCLUSÕES}

A frequência de retinopatia da prematuridade nos recémnascidos do HCFMRP-USP foi de 35,71\% entre os pré-termos com peso menor que 1.500 gramas e de $1,36 \%$ no total de nascimentos no período de fevereiro de 2005 a janeiro de 2006. Os fatores de risco associados à doença no estudo foram: peso, idade gestacional; escore SNAPPE II, uso de oxigenoterapia por ventilação mecânica e CPAP, múltiplas transfusões sanguíneas e o uso de diuréticos. Foi encontrada a associação inversa entre a imaturidade retiniana e o estágio de ROP sendo constatado um aumento de casos no estágio III, grupo que apresentava a menor idade gestacional e peso.

\section{ABSTRACT}

Purpose: To determine the frequency of retinopathy of prematurity at the Clinical Hospital of Ribeirão Preto Medical School (HCFMRP-USP) and verify the association of retinopathy of prematurity with known risk factors. Methods:
Prospective analysis of 70 patients born in the HCFMRP-USP, weighing less than 1,500 grams, in a period of 1 year. The patients were divided into 2 groups (Retinopathy of prematurity and Normal) to verify the association of retinopathy of prematurity and known risk factors. Significance level of 5\% was used. Results: The frequency of retinopathy of prematurity was $35.71 \%$ among the study population. The studied risk factors significantly related to the development of the disease were: weight $(\mathrm{p}=0.001)$, gestational age $(\mathrm{p}=0.001)$, score SNAPPE II $(\mathrm{p}=0.008)$, use of oxygen by intubation $(\mathrm{p}=0.019)$ and continuous positive airway pressure $(\mathrm{p}=0.0017)$, multiple blood transfusions $(\mathrm{p}=0.01)$, and the use of diuretics $(\mathrm{p}=0.01)$. Conclusion: The frequency of retinopathy of prematurity was $35.71 \%$ and several risk factors have been identified in newborns in the HCFMRP-USP, being evidenced that the more preterm, the more severe retinopathy of prematurity is likely to be.

Keywords: Retinopathy of prematurity/epidemiology; Infant, newborn; Risk factors; Retrospective studies; Gestational age; Birth weight

\section{REFERÊNCIAS}

1. Terry TL, Fibroblastic overgrowth of persistent tunica vasculosa lentis in infants born prematurely: II. Report of cases-clinical aspects. Trans Am Ophthalmol Soc. 1942;40:262-84

2. Silverman W. Retrolental fibroplasia: a modern parable. New York: Grune \& Stratton; 1980. p.264.

3. Wheatley CM, Dickinson JL, Mackey DA, Craig JE, Sale MM. Retinopathy of prematurity: recent advances in our understanding. Br J Ophthalmol, 2002; 86(6):696-700.

4. Lad EM, Nguyen TC, Morton JM, Moshfeghi DM. Retinopathy of prematurity in the United States. Br J Ophthalmol. 2008;92(3):320-5.

5. Chiang MF, Arons RR, Flynn JT, Starren JB. Incidence of retinopathy of prematurity from 1996 to 2000: analysis of a comprehensive New York state patient database. Ophthalmology. 2004;111(7):1317-25. Comment in: Ophthalmology. 2005;112(4):738; author reply 738-9.

6. Gilbert C, Rahi J, Eckstein M, O’Sullivan J, Foster A. Retinopathy of prematurity in middle-income countries. Lancet. 1997;350(9070):12-4.

7. Gilbert C. Retinopathy of prematurity: a global perspective of the epidemics, population of babies at risk and implications for control. Early Hum Dev. 2008;84(2):77-82. Review.

8. Graziano RM, Leone CR, Cunha SL, Pinheiro AC. [Prevalence of retinopathy of prematurity in very low birth weight infants]. J Pediatr (Rio J). 1997;73(6): 377-82. Portuguese.

9. Alves MAS, Analise dos fatores de risco para o desenvolvimento da retinopatia da prematuridade. Rev Bras Oftalmol. 1995;54(10):735-49.

10. Fortes Filho JB, Eckert GU, Procianoy L, Barros CK, Procianoy RS. Incidence and risk factors for retinopathy of prematurity in very low and in extremely low birth weight infants in a unit-based approach in southern Brazil. Eye (Lond). 2009;23(1):25-30

11. Zin A, Florêncio T, Fortes Filho JB, Nakanami CR, Gianini N, Graziano RM, Moraes N; Brazilian Society of Pediatrics, Brazilian Council of Ophthalmology and Brazilian Society of Pediatric Ophthalmology. [Brazilian guidelines proposal for screening and treatment of retinopathy of prematurity (ROP)]. Arq Bras Oftalmol. 2007;70(5):875-83.

12. Hammer ME, Mullen PW, Ferguson JG, Pai S, Cosby C, Jackson KL. Logistic analysis of risk factors in acute retinopathy of prematurity. Am J Ophthalmol. 1986;102(1):1-6.

13. Lermann VL, Fortes Filho JB, and Procianoy RS, The prevalence of retinopathy of prematurity in very low birth weight newborn infants. J Pediatr (Rio J), 2006;82(1): 27-32.

14. Tasman W, Patz A, McNamara JA, Kaiser RS, Trese MT, Smith BT. Retino- 
pathy of prematurity: the life of a lifetime disease. Am J Ophthalmol. 2006; 141(1):167-74

15. Lima DRR, Estudo da ocorrência e evolução da retinopatia da prematuridade e fatores associados no Hospital das Clínicas da Faculdade de Medicina de Ribeirão Preto - USP. Ribeirão Preto: Universidade de São Paulo: 2006

16. An international classification of retinopathy of prematurity. II. The classification of retinal detachment. The International Committee for the Classification of the Late Stages of Retinopathy of Prematurity. Arch Ophthalmol. 1987; 105(7):906-12. Erratum in: Arch Ophthalmol. 1987;105(11):1498.

17. Segundo Workshop de Retinopatia da Prematuridade. Rio de Janeiro: Instituto Brasileiro de Oftalmologia, dez 3-4, 2004. [citado 2009 Jan 10]. Disponível em: http://www.cbo.com.br/cbo/sociedades/pediatria/artigos/RelatorioIIWorkshop ROP.pdf

18. Palmer EA, Flynn JT, Hardy RJ, Phelps DL, Phillips CL, Schaffer DB, et al Incidence and early course of retinopathy of prematurity. The Cryotherapy for Retinopathy of Prematurity Cooperative Group. Ophthalmology. 1991;98(11): 1628-40.

19. Good WV, Hardy RJ, Dobson V, Palmer EA, Phelps DL, Quintos M, Tung B; Early Treatment for Retinopathy of Prematurity Cooperative Group. The incidence and course of retinopathy of prematurity: findings from the early treatment for retinopathy of prematurity study. Pediatrics. 2005;116(1):15-23.

20. Bassiouny MR, Risk factors associated with retinopathy of prematurity: a study from Oman. J Trop Pediatr. 1996;42(6):355-8.

21. Grunauer N, Iriondo Sanz M, Serra Castanera A, Krauel Vidal J, Jimenex
Gonzales R. [Retinopathy of prematurity: casuistics between 1996 and 2001]. An Pediatr (Barc). 2003;58(5):471-7.

22. Fortes Filho JB, Dill JC, Ishizaki A, Aguiar WW, Silveira RC, Procianoy RS. Score for Neonatal Acute Physiology and Perinatal Extension II as a predictor of retinopathy of prematurity: study in 304 very-low-birth-weight preterm infants. Ophthalmologica. 2009;223(3):177-82.

23. Supplemental Therapeutic Oxygen for Prethreshold Retinopathy Of Prematurity (STOP-ROP), a randomized, controlled trial. I: primary outcomes. Pediatrics. 2000;105(2):295-310. Comment in: Pediatrics. 2002;110(3):615-6. Pediatrics. 2000;1-5(2):420-1. Pediatrics. 2000;105(2):424-5.

24. Higgins RD, Mendelsohn AL, DeFeo MJ, Ucsel R, Hendricks-Munoz KD. Antenatal dexamethasone and decreased severity of retinopathy of prematurity. Arch Ophthalmol. 1998;116(5):601-5.

25. Hussain N, Clive J, Bhandari V, Current incidence of retinopathy of prematurity, 1989-1997. Pediatrics. 1999;104(3):e26

26. Yanovitch TL, Siatkowski RM, McCaffree M, Corff KE. Retinopathy of prematurity in infants with birth weight $>$ or $=1,250$ grams-incidence, severity, and screening guideline cost-analysis. J AAPOS. 2006;10(2):128-34.

27. Jandeck C, Kellner U, Kossel H, Bartsch M, Versmold HT, Foerster MH Retinopathy of prematurity in infants of birth weight $>2,000 \mathrm{~g}$ after haemorrhagic shock at birth. Br J Ophthalmol. 1996;80(8):728-31.

28. Hirano K, Morinobu T, Kim H, Hiroi M, Ban R, Ogawa S, et al., Blood transfusion increases radical promoting non-transferrin bound iron in preterm infants. Arch Dis Child Fetal Neonatal Ed. 2001;84(3):F188-93.

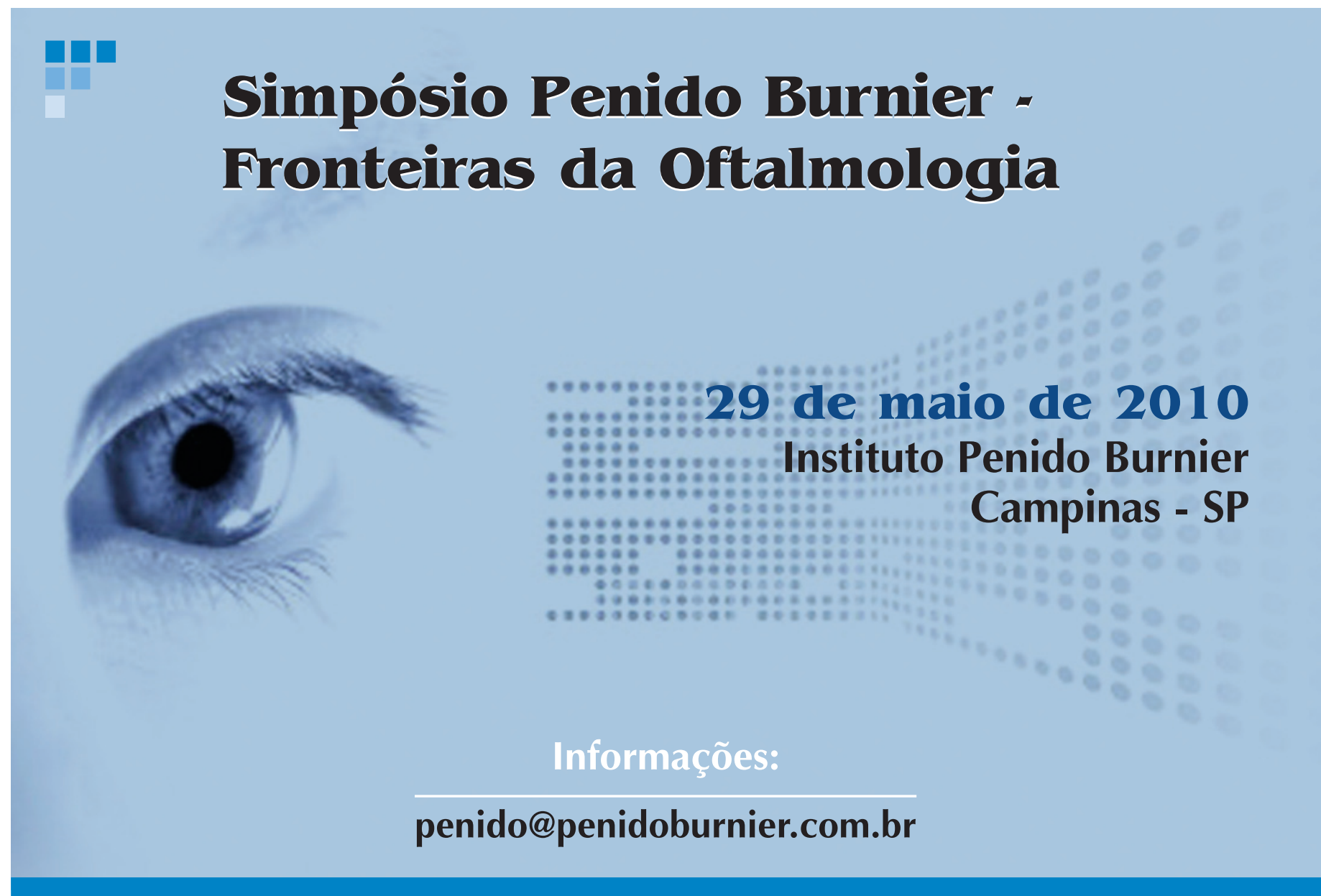

